\title{
COMMENTARY
}

\section{A Call to Action for Cultural Humility in Pharmacy Education Student Assessments}

\author{
Denise Rizzolo, $\mathrm{PhD},{ }^{\mathrm{a}}$ Chadwin Sandifer, EdD, ${ }^{\mathrm{b}}$ Julie Kalabalik-Hoganson PharmD, ${ }^{\mathrm{b}}$ Nora Lowy, $\mathrm{PhD}{ }^{\mathrm{b}}$ \\ ${ }^{a}$ Fairleigh Dickinson University, Master of Public Health, Madison, New Jersey \\ ${ }^{\mathrm{b}}$ Fairleigh Dickinson University, School of Pharmacy \& Health Sciences, Madison, New Jersey
}

Corresponding Author: Denise Rizzolo, Fairleigh Dickinson University, Master of Public Health, 230 Park Ave, Madison, NJ 07940. Tel: 973-443-8235. Email: drizzolo@fdu.edu

Submitted March 4, 2021; accepted June 2, 2021; ePublished June 2021

An important topic in conversation on the education of pharmacy students has evolved around methods of pedagogy and assessment, and attention to diversity and inclusion. Well-intentioned educators may introduce bias into their teachings and assessment tools by focusing on diseases with a higher rate of presentation in minorities, without engaging in conversations as to why these health disparities may exist. When considering the content and structure of a curriculum, it is also important to review its assessment tools, with attention to cultural humility in multiple-choice examinations, case-based presentations and even observed structured clinical examinations. Disregarding this component of the conversation may lead students to have an unconscious impression that social constructs are biological markers for a disease. Students will recall not only what they learned in a classroom setting, but often the content included in their assessments as well. By writing test items that are culturally responsible, unconscious bias can be reduced and test items can better measure the knowledge that educators intend to assess. As pharmacy educators perform programmatic reviews, attention should be directed towards unconscious bias not only in the curricula but also in its evaluation and assessment tools.

Keywords: cultural humility, pharmacy assessment, test-items, diversity, inclusion, unconscious bias

\section{INTRODUCTION}

In pharmacy education, conversations have recently focused on diversity and inclusion within methods of pedagogy and assessment. Considering recent social and racial injustices in the United States over the past year, pharmacy organizations have joined the conversation about diversity, inclusion, and equity in the profession and pharmacy curricula. On June 5, 2020, 14 national pharmacy organizations, including the American Association of Colleges of Pharmacy (AACP), issued a joint statement on the need to address racism and discrimination and to take action to promote social justice and address diversity, equity, and inclusion. ${ }^{1}$ As an organization, AACP values inclusiveness and developed the Equity, Diversity, and Inclusion Institute to promote the advancement of these elements at AACP member institutions. The Accreditation Council for Pharmacy Education (ACPE) appointed an Equity, Diversity and Inclusion Subcommittee to the Board with the goal of updating the Guidance for Standards 2016 with regards to equity, diversity, and inclusion. ${ }^{2}$ As the profession focuses attention on these important areas, pharmacy educators are encouraged to reflect on what is called the "silent curriculum" and how this unconscious bias may influence students' thoughts, feelings, and actions. ${ }^{3}$ Unconscious bias describes associations or attitudes that reflexively alter our perceptions, thereby affecting behavior, interactions, and decision-making. ${ }^{4}$

At times, well-intentioned educators may introduce bias into their teachings and assessments by focusing on diseases that have a higher rate in minorities, without engaging in conversations as to why these health disparities may exist or examine them through social determinants of health. ${ }^{3}$ Disregarding these critical components of the conversation may lead students to an unconscious impression that certain diseases may always impact certain groups and subgroups more than others. This type of unconscious bias may also influence quality of care (among other factors).

ACPE Standards include the need for students to demonstrate cultural awareness and sensitivity. ${ }^{2}$

While curricula are reviewed to ensure they are inclusive of cultural competency, to date, assessment tools including multiple choice examinations, clinical presentations, and objective structured clinical examinations (OSCEs) have not been subjected to the same scrutiny. Research in the area of unconscious bias in medical education assessment tools is limited and almost none exists within pharmacy education. ${ }^{5}$ While pharmacy and other health care educators are aware of how teaching can lead to unconscious bias, the question remains as to whether student assessments lead to unconscious bias. Previous research has illustrated that unconscious bias exists in medical school assessment tools, but 
whether it exists in pharmacy assessments remains unknown. Therefore, it is worthy of further exploration with the goal of ensuring culturally sensitive pharmacy assessments tools.

Cultural humility has been defined as a "process of being aware of how people's culture can impact their health behaviors and in turn using this awareness to cultivate sensitive approaches in treating patients". ${ }^{6}$ Frameworks to integrate cultural humility into curricula have been described but have yet to be implemented and evaluated. Rockich-Winston and Wyatt described the essential elements of teaching using a blended model of cultural humility and cultural competency called culturally responsive teaching; however, literature in the area of cultural humility in pharmacy assessment is lacking. ${ }^{7}$

An important concern in health care involves the disparities associated with certain diseases. These distributions may be attributable to socioeconomic factors and teaching should be focused around these factors that could be contributing to the disparities. Although the contributions of socioeconomic variables to disease etiology and progression have been acknowledged, the possibility of implicit bias in clinical practice must also be considered. For health care educators, this raises questions about how best to teach about diseases with genetic predispositions and/or greater incidences in certain racial or ethnic groups and how to construct assessment tools that are unbiased in their inclusion of race and ethnicity. This question is especially important when considering multiple choice question assessment tools.

The research examined thus far on unconscious bias focuses on curricula and very little has examined assessment. A study by Ripp and Braun analyzed a bank of questions used by medical students in preparation for the United States Medical Licensing Examination (USMLE). ${ }^{5}$ The study reviewed 2,211 questions purchased from the World Step 1 QBank (a test bank for the USMLE), identified questions that referred to race/ethnicity $(n=455,20.6 \%)$, quantified the number of mentions of race/ethnicity $(n=474)$, and categorized those mentions as either descriptive $(86.9 \%)$ or central $(20.6 \%)$ to the case. An analysis of the 455 questions mentioning race/ethnicity revealed that for almost all vignettes $(90.5 \%)$, race/ethnicity was simply a demographic descriptor with no central relevance to case content. In addition, when comparing questions by ethnicity, questions mentioning Whites/Caucasians were distributed across the bank at a significantly higher rate than other ethnicities/races $(p>.001)$ at a rate disproportionate to the U.S. population: $85.8 \%$ of the questions referred to White/Caucasians, $9.7 \%$ to Black/African Americans, $3.16 \%$ to Asians, $0.633 \%$ to Hispanics, and $0.633 \%$ to Native Americans. In addition, whether the use of race was descriptive or central to the case varied considerably. For questions mentioning Blacks/African Americans, of the three most common diseases included in the cases, only one (Sickle cell) was correlated to genetics, while the other two conditions (coronary heart disease and hypertension) were not related to genetics but rather environmental and lifestyle factors. ${ }^{5}$ While sickle cell disease may be most common in African Americans, it does occur amongst Hispanic-Americans as well. Linking one race consistently to a specific disease may lead students to exclude that potential diagnosis from their differential with other patients. For example, although sickle cell disease may be most common in African Americans, it may also need to be considered in Hispanic-Americans. The authors of the study, therefore, theorized the routine use of race/ethnicity with no specific goal in preparation of materials, such as question banks, risks contributing to racial bias. Ripp and Braun suggest that the item vignettes should be reflective of the national distribution. ${ }^{5}$

Additionally, a study by Nawaz and Brett attempted to determine whether U.S. medical students were being taught to mention race at the beginning of case presentations. ${ }^{9}$ The authors surveyed 122 medical schools with a response rate of $85 \%$ (104/122). Responses from medical schools indicated that only $11 \%$ of schools explicitly taught students to mention race at the beginning of case presentations, $63 \%$ of schools selectively taught students to include race in the first sentence, while only $9 \%$ of schools responded that the practice was discouraged. In addition, students claimed that physicians mentioned race at the start of their presentations during clinical rounds. ${ }^{9}$ Clinical presentations are similarly part of pharmacy education, both in the didactic and experiential settings. When students are encouraged to routinely include race without it being central to the case, this practice may lead to unconscious bias. ${ }^{9}$

To date, research on unconscious bias in assessment has been limited to medical education and currently no focus has been noted on investigating pharmacy assessment tools. As assessment tools evolve in consideration of cultural humility, pharmacy educators should begin to evaluate their test items and begin to explore their own item writing practices and whether current practices may be leading to unconscious bias.

While medical educators are reviewing their assessments for cultural humility, pharmacy educators should also be examining their assessment tools to ensure they are yielding their intended purposes. For example, if every question item on the topic of sexually transmitted infections (STI) begins with the opening sentence "An African American male with a history of STI", it could lead students to believe STIs are infections that predominately occur in African American males, thus adding to an unconscious systemic racism. Educators should consider following the item writing guidelines issued by the National Board of Medical Examiners. ${ }^{10}$ 
The recommendation is that race and/or ethnicity be defined by the patient and not the provider, included when relevant, and not necessarily placed in the opening sentence. ${ }^{10}$ In item creation, patient characteristics should be included in the vignettes if they add to the understanding of the case and should never contain negative stereotypes. ${ }^{10}$ It is not being suggested that race be removed completely from all vignettes, but rather that race be either self-reported by patients or included in vignette when clinically appropriate. ${ }^{10}$

\section{CONCLUSION}

The Accreditation Council for Pharmacy Education and other pharmacy related organizations such as the American College of Clinical Pharmacy (ACCP), the American Pharmacists Association (APhA), and American Society of Health-System Pharmacists (ASHP) have continued to promote and support cultural humility during the preprofessional education of pharmacy students and professional practice of pharmacists. Pre-professional education provides a foundation for culturally competent practicing pharmacists by incorporating into the curriculum diverse and inclusive perspectives regarding patient care. It is suggested that educators examine their curricula for cultural diversity while also reviewing their assessments to ensure they do not unintentionally lead to bias. Race and ethnicity are not irrelevant to pharmacy education but should be taught and referred to in assessment tools in ways that do not perpetuate unconscious bias. ${ }^{11}$ Encouraging faculty self-reflection on the influences of unconscious bias is recommended. Additionally, faculty training workshops on item writing and development are needed in order to evaluate current tools and practices that minimize unconscious bias in pharmacy assessment.

\section{REFERENCES}

1. Pharmacy Organizations Unite to Take a Stand Against Racial Injustice. Update: National Pharmacy Organizations Take Action Against Racial Injustices. American Society of Health-System Pharmacists; 2020. Available at: https://www.ashp.org/-/media/assets/news-and-media/docs/Pharmacy-Groups-StatementPharmacists-RacialInjustice. ashx?la=en\&hash=6310D7371D0C91E67A8A79B34D096017596AC67C . Accessed March 1, 2021.

2. Accreditation Standards and Key Elements for the Professional Program in Pharmacy Leading to the Doctor of Pharmacy Degree. Accreditation Council for Pharmacy Education; 2015. Available at: https://www.acpeaccredit.org/pdf/Standards2016FINAL.pdf.

3. Accessed February 25, 2021.

4. Katherine C. Brooks BA. A Silent Curriculum. JAMA. https://jamanetwork.com/journals/jama/articleabstract/2293299. Published May 19, 2015. Accessed February 25, 2021.

5. Marcelin JR, Siraj DS, Victor R, Kotadia S, Maldonado YA. The impact of unconscious bias in healthcare: How to recognize and mitigate it. J Infect Dis. 2019;20;220(220 Suppl 2):S62-S73. doi: 10.1093/infdis/jiz214. PMID: 31430386.

6. Ripp K, Braun L. Race/ethnicity in medical education: an analysis of a question bank for step 1 of the United States Medical Licensing Examination. Teach Learn Med. 2017;29(2):115-22.

7. Prasad SJ, Nair P, Gadhvi K, Barai I, Danish HS, Philip AB. Cultural humility: treating the patient, not the illness. Med Educ Online. 2016;21:30908. Published 2016 Feb 3. doi:10.3402/meo.v21.30908

8. 7.Rockich-Winston N, Wyatt TR. The case for culturally responsive teaching in pharmacy curricula. Am J Pharm Educ. 2019;83(8):7425. doi:10.5688/ajpe7425.

9. 8.Centers of Disease Control and Prevention (CDC) Data and Statistics on Sickle Cell Disease. December 16, 2020. Accessed May 21,

2021. https://www.cdc.gov/ncbddd/sicklecell/data.html\#: :text=Sickle\%20cell\%20disease\%20(SCD)\%20affects, and $\% 20$ Mediterranean\%20countries\%20such\%20as

10. Nawaz H, Brett A. Mentioning race at the beginning of clinical case presentations: a survey of US medical schools. Med Educ. 2009;43(2):146-154.

11. Item writing guide. National Board of Medical Examiners. 2020. https://www.nbme.org/item-writing-guide. Accessed March 3, 2021.

12. Amutah C, Greenidge K, Mante A, Munyikwa M, Surya SL, Higginbotham E. Misrepresenting Race - The Role of Medical Schools in Propagating Physician Bias: NEJM. New England Journal of Medicine. https://www.nejm.org/doi/full/10.1056/NEJMms2025768. Published January 6, 2021. Accessed March 4, 2021. 